\title{
UAV Based Agricultural Crop Canopy Mapping for Crop Field Monitoring
}

\author{
Prabath Priyankara $^{\mathrm{a}, \mathrm{b}, *}$, Takehiro Morimoto ${ }^{\mathrm{c}}$ \\ ${ }^{a}$ Graduate School of Life and Environmental Sciences, University of Tsukuba, 1-1-1, Tennodai, Tsukuba, Ibaraki 305-8572, Japan, \\ prabath@geoenv.tsukuba.ac.jp \\ ${ }^{b}$ Faculty of Agriculture, University of Ruhuna, Mapalana, Kamburupitiya 81100, Sri Lanka \\ ${ }^{c}$ Faculty of Life and Environmental Sciences, University of Tsukuba, 1-1-1, Tennodai, Tsukuba, Ibaraki 305-8572, Japan, \\ tmrmt@geoenv.tsukuba.ac.jp \\ * Corresponding author
}

Keywords: Agricultural Crop, Crop Canopy Mapping, Crop Field Monitoring, UAV

\begin{abstract}
:
Nowadays, mapping of agricultural crop canopy in different growing stages are vital data for crop field monitoring than field-based observations in large scale agricultural crop fields. By mapping agricultural crop canopy, it is very easy to analyse the status of an agricultural crop field by using different vegetation indices. Further, the data can be used to estimate the yield. These information are timely and reliable spatial information to the farmers and decision makers. Mapping of crop canopy in an agricultural crop field in different growing stages are very challenging using satellite imagery mainly due to the difficulty of recording with high cloud coverage. Also, the cost for satellite imagery are higher in proportion to the spatial resolution. It takes some time to order a satellite imagery and sometimes can't cover some growing stages. This problem can be solved by using low cost RGB based UAV imageries which can be operated at low altitudes (below the clouds) which and when necessary. This study is therefore aimed at mapping of a maize crop canopy using RGB based UAV imageries. UAV flights at different growth stages were carried out with a high resolution RGB camera over a maize field in Ampara District, Sri Lanka. For accurate crop canopy mapping, very high-resolution multitemporal ortho-mosaicked images were derived from UAV imageries using free and open source image processing platforms with spatial resolution in centimetre level. The resultant multi-temporal ortho-mosaicked images can be used to map and monitor the crop field's precise and efficient manner. These information are very important for farmers and decision makers to properly manage the crop fields.
\end{abstract}

